\title{
THIN COHERENT CONFIGURATIONS AND GROUPOIDS
}

\author{
AKIHIDE HANAKI AND MASAYOSHI YOSHIKAWA
}

\begin{abstract}
In the present paper, we will investigate thin coherent configurations. We will show that thin coherent configurations are equivalent to finite connected groupoids. Also, we will investigate their representations.
\end{abstract}

coherent configurations; association schemes; thin; finite groups; finite connected groupoids

\section{INTRODUCTION}

In [6], Higman defined coherent configurations in order to study permutation groups. Recently, coherent configurations has studied for applying to the theory of association schemes which are the generalization of permutation groups. Thus, we have the motivation of studying the coherent configurations themselves.

It is well-known that thin association schemes are essentially finite groups. K. Kuribayashi, a topologist, asked to authors that what objects are corresponding to groupoids. Our answer for this question is that thin coherent configurations are essentially finite connected groupoids. In order to show this, we will determine the structure of thin coherent configurations. It seems to be well-known, but we will give a complete proof because we could not find a good reference.

As the result, we will obtain that the representations of the thin coherent configuration are Morita equivalent to the representations of the vertex group.

\section{Preparation}

All statements in this section seem to be known, but we will give their proofs for the readers.

2.1. Coherent Configurations. For definitions in the present subsection, refer to [6].

If $X$ is a finite non-empty set and $C$ is a set of non-empty binary relations on $X$, so that $C$ is a subset of the power wet $\mathfrak{P}\left(X^{2}\right)$ of the Cartesian square of $X$, then we call $(X, C)$ the configuration based on $X$ with $C$ as its set of basic relations. We call $n=|X|$ the degree and $r=|C|$ the rank. 
If $R$ is a commutative ring and $X$ is finite non-empty set, we write $\operatorname{Mat}_{X}(R)$ for the $R$-module of matrices with coefficients in $R$ having rows and columns indexed by $X$. We regarded $\operatorname{Mat}_{X}(R)$ as a $R$-algebra with respect to matrix multiplication.

For $c \subseteq X^{2}, A_{c}$ will denote the adjacency matrix of the directed graph $(X, c)$. Thus $A_{c} \in \operatorname{Mat}_{X}(\mathbb{Z})$ and $\left(A_{c}\right)_{x y}=1$ or 0 according as $(x, y) \in c$ or $X^{2}-c$.

We call a configuration satisfying the following axioms (I) through (IV) coherent,

(I) $C$ is a partition of $X^{2}$.

(II) If $f \in C$ and $f \cap I_{X} \neq \emptyset$, where $I_{X}=\{(x, x) \mid x \in X\}$, then $f \subseteq I_{X}$.

(III) $c \in C$ implies $c^{*}=\{(y, x) \mid(x, y) \in c\} \in C$.

(IV) For $a, b, c \in C$ and $(x, y) \in c$, the integer $p_{a b}^{c}=|x a \cap b y|$ is independent of the choice of $x$ and $y$, where for $x \in X$ and $c \in C, x c=\{y \in X \mid(x, y) \in c\}$ and $c x=\{y \in X \mid(y, x) \in c\}$.

We set that $\mathbb{B}=\left\{A_{c} \mid c \in C\right\}$. In terms of the adjacency matrices, these four axiom is equivalent to

(I) $\sum_{c \in C} A_{c}=J$, where $J$ is all one matrix of degree $|X|$.

(II) there is the subset $C_{0}$ of $C$ such that $\sum_{f \in C_{0}} A_{f}=I$, where $I$ is the identity matrix of order $|X|$.

(III) $A_{c^{*}}={ }^{t} A_{c} \in \mathbb{B}$ for any $c \in C$, where ${ }^{t} M$ is the transpose of the matrix $M$.

(IV) For $a, b, c \in C$, there are integers $p_{a b}^{c}$ such that $A_{a} A_{b}=\sum_{c \in C} p_{a b}^{c} A_{c}$.

We call the element of the subset $C_{0}$ the fiber and integers $p_{a b}^{c}$ the intersection numbers. From the axiom (IV), for any commutative ring $R$ with the identity, we can define the $R$-algebra $R C$ as the subalgebra of the matrix algebra $\operatorname{Mat}_{X}(R)$;

$$
R C=\bigoplus_{c \in C} R A_{c}
$$

We call the $R$-algebra $R C$ the adjacency algebra of the coherent configuration $(X, C)$ over the ring $R$.

For the rest of the present paper, we assume that $(X, C)$ is coherent.

We will introduce three special configurations. First, if we have that $C=\left\{I_{X}, X^{2}-I_{X}\right\}$, we say that the coherent configuration $(X, C)$ is trivial. Secondly, if we have that $C$ is the partition of $X^{2}$ into singleton sets, we call it discrete. Finally, if we have that $I_{X} \in C$, we say that the coherent configuration $(X, C)$ is homogeneous. Essentially, homogeneous coherent configurations are equivalent to association schemes, where we consider that association schemes are not necessary commutative. For association schemes, refer to [1], [7].

Let $(X, C)$ be a coherent configuration and $f \in C$ be a fiber. We set that $X_{f}=\{x \in X \mid(x, x) \in f\}$ and $C_{f}=\left\{c \cap X_{f}^{2} \mid c \in C\right\}$. 
Then, the pair $\left(X_{f}, C_{f}\right)$ become the coherent configuration. Especially, for any fiber $f$, the coherent configuration $\left(X_{f}, C_{f}\right)$ is homogeneous. We call the coherent configuration $\left(X_{f}, C_{f}\right)$ the fiber scheme of the configuration $(X, C)$ by the fiber $f$.

Let $(X, C)$ and $(Y, D)$ be two coherent configurations. We set that $Z=X \times Y$ and $E=\{(c, d) \mid c \in C, d \in D\}$, where $((x, y),(z, w)) \in(c, d)$ implies $(x, z) \in c,(y, w) \in d$. Thus, we can define a new coherent configuration $(Z, E)$ from $(X, C)$ and $(Y, D)$. This new coherent configuration $(Z, E)$ is called the direct product of the configurations $(X, C)$ and $(Y, D)$. Then we have that $A_{(c, d)}=A_{c} \otimes A_{d}$ for all $c \in C, d \in D$, where $\otimes$ means the Kronecker's product. Therefore, it follows that $R E=R C \otimes_{R} R D$ for any commutative ring $R$ with the identity.

We will introduce some properties for the coherent configuration.

Lemma 1. Let $(X, C)$ be a coherent configuration and $C_{0}$ be the set of fibers of $(X, C)$. Then, it follows the followings.

(1) There exists the unique fiber $f \in C_{0}$ for any $c \in C$ such that $p_{f c}^{c}=1$. Moreover, we have that $p_{g c}^{c}=0$ for any $g \in C_{0}$ with $g \neq f$.

(2) There exists the unique fiber $f \in C_{0}$ for any $c \in C$ such that $p_{c f}^{c}=1$. Moreover, we have that $p_{c g}^{c}=0$ for any $g \in C_{0}$ with $g \neq f$.

Proof. We set that $(x, y) \in c$ and $f \in C_{0}$. If $x \notin X_{f}, x f=\emptyset$. Therefore, we have that $p_{f c}^{c}=0$. If $x \in X_{f}$, we obtain that $p_{f c}^{c}=1$. Thus the fact (1) follows. Similarly, we have the fact (2).

We set that for any $f, g \in C_{0}$,

$$
\begin{aligned}
{ }_{f} C & =\left\{c \in C \mid p_{f c}^{c}=1\right\}, \\
C_{g} & =\left\{c \in C \mid p_{c g}^{c}=1\right\}, \\
{ }_{f} C_{g} & =\left\{c \in C \mid p_{f c}^{c}=p_{c g}^{c}=1\right\} .
\end{aligned}
$$

Then, we have the following three partitions of $C$ from Lemma 1 :

$$
C=\bigcup_{f \in C_{0}}{ }_{f} C=\bigcup_{g \in C_{0}} C_{g}=\bigcup_{f, g \in C_{0}} C_{g} .
$$

Moreover, we have the following lemma.

Lemma 2. We obtain that $c \in{ }_{f} C$ if and only if $c^{*} \in C_{f}$, and $c \in{ }_{f} C_{g}$ if and only if $c^{*} \in{ }_{g} C_{f}$.

Proof. By the direct calculation, it follows that

$$
c \in{ }_{f} C \Leftrightarrow p_{f c}^{c}=1 \Leftrightarrow 1=|x f \cap c y| \text { for some }(x, y) \in c .
$$

Then, we have that $1=\left|y c^{*} \cap f^{*} x\right|$ for $(y, x) \in c^{*}$. Namely, we obtain that $p_{c^{*} f^{*}}^{c^{*}}=1$. However, we know that $f=f^{*}$ since $f$ is a fiber. Therefore, it follows that $c^{*} \in C_{f}$. In the latter half, we have the desiring result, similarly. 
Lemma 3. Let $f, g, h, i, j, k \in C_{0}$ be fibers.

For $a \in{ }_{f} C_{g}, b \in{ }_{h} C_{i}, c \in{ }_{j} C_{k}$, we have that $p_{a b}^{c}=0$ if $g \neq h, f \neq j$ or $i \neq k$.

For $c \in{ }_{f} C_{g}$, we define the out-valency $n_{c}$ and the in-valency $n$ of $c$ by $n_{c}=p_{c c^{*}}^{f},{ }_{c} n=p_{c^{*} c}^{g}$, respectively. We have obviously that $n_{c}={ }_{c^{*}} n$ for any $c \in C$. For $x \in X_{f}$, we obtain that $|x c|=\left|x c \cap c^{*} x\right|=p_{c c^{*}}^{f}=n_{c}$. Similarly, it follows that $|c y|={ }_{c} n$ for $y \in X_{g}$. Therefore, we have that $\left|X_{f}\right| n_{c}=|c|=\left|X_{g}\right|_{c} n$. Considering the adjacency matrix $A_{c}$, these facts mean that each column of $A_{c}$ corresponding to the element of $X_{f}$ has $n_{c} 1$ 's and each row of $A_{c}$ corresponding the element of $X_{g}$ has ${ }_{c} n$ 1 's. From the definition, we have that

$$
\sum_{c \in f} n_{c}=|X|, \sum_{c \in C_{g}}{ }_{c} n=|X|, \sum_{c \in f_{f} C_{g}} n_{c}=\left|X_{g}\right|, \sum_{c \in \in_{f} C_{g}}{ }_{c} n=\left|X_{f}\right| .
$$

Lemma 4. For $a, b, c \in C$, it follows that $|a| p_{b c}^{a^{*}}=|b| p_{c a}^{b^{*}}=|c| p_{a b}^{c^{*}}$.

Proof. From the associative law on the multiplication, for $a, b, c, d \in C$, we have

$$
\sum_{s} p_{a b}^{s} p_{s c}^{d}=\sum_{t} p_{b c}^{t} p_{a t}^{d}
$$

We set that $a \in{ }_{f} C_{g}, b \in{ }_{g} C_{h}, c \in{ }_{h} C_{f}$. By Lemma 3, we know that $p_{b c}^{a^{*}}=p_{c a}^{b^{*}}=p_{a b}^{c^{*}}=0$ out of this setting. Therefore it follows the desiring formula. If we set that $d=f$ in the above formula, the left side is

$$
\sum_{s} p_{a b}^{s} p_{s c}^{f}=p_{a b}^{c^{*}} p_{c^{*} c}^{f}={ }_{c} n p_{a b}^{c^{*}},
$$

and the right side is

$$
\sum_{t} p_{b c}^{t} p_{a t}^{f}=p_{b c}^{a^{*}} p_{a a^{*}}^{f}=n_{a} p_{b c}^{a^{*}} .
$$

Then, if we multiply both sides by $\left|X_{f}\right|$, we have that $|a| p_{b c}^{a^{*}}=|c| p_{a b}^{c^{*}}$. Other cases are proved by the similar way.

Finally, we list the useful formulas. This list may have crossover.

Proposition 5. Let $f, g, h \in C_{0}$ be fibers.

(1) $p_{c h}^{c}=\delta_{g h}, p_{h c}^{c}=\delta_{f h}, p_{c g}^{d}=\delta_{c d}, p_{f c}^{d}=\delta_{c d}$ for $c, d \in{ }_{f} C_{g}$,

(2) $|x c|=n_{c}$ for $x \in X_{f}, c \in{ }_{f} C$,

(3) $\left|X_{f}\right| n_{c}=|c|=\left|X_{g}\right|_{c} n$ for $c \in{ }_{f} C_{g}$,

(4) $\sum_{c \in_{f} C} n_{c}=|X|, \sum_{c \in C_{g}} n=|X|$,

(5) $\sum_{c \in_{f} C_{g}} n=\left|X_{g}\right|, \sum_{c \in C_{f} C_{g}} n_{c}=\left|X_{f}\right|$,

(6) $|a| p_{b c}^{a^{*}}=|b| p_{c a}^{b^{*}}=|c| p_{a b}^{c^{*}}$ for $a, b, c \in C$,

(7) $\sum_{b \in{ }_{g} C_{h}} p_{a b}^{c}=\sum_{b \in C} p_{a b}^{c}=n_{a}$ for $a \in{ }_{f} C_{g}, c \in{ }_{f} C_{h}$,

(8) $\sum_{b \in{ }_{h} C_{f}} p_{b a}^{c}=\sum_{b \in C} p_{b a}^{c}={ }_{a} n$ for $a \in{ }_{f} C_{g}, c \in{ }_{h} C_{g}$. 
Proof. We know already that the facts from (1) to (6) hold. We will prove the fact (7). We define the matrix $J_{f g} \in \operatorname{Mat}_{X}(\mathbb{Z})$ as follows. If $x \in X_{f}$ and $y \in X_{g}$, the $(x, y)$-entry of $J_{f g}$ is 1 . Otherwise the $(x, y)$-entry of $J_{f g}$ is 0 . Namely, we have that $J_{f g}=\sum_{c \epsilon_{f} C_{g}} A_{c}$. Then, if $c \in{ }_{f} C_{g}$, it follows that $A_{c} J_{g h}=n_{s} J_{f h}$. It means that the fact (7) holds. The fact (8) is proved by the similarly way.

2.2. finite connected groupoids. For definitions in the present subsection, refer to [5].

The small category $\mathfrak{G}$ is called a groupoid if every morphism is invertible. Namely, every $f \in \operatorname{Mor}_{\mathfrak{G}}(X, Y)$ has an inverse $f^{-1} \in \operatorname{Mor}_{\mathfrak{G}}(Y, X)$ with $f \circ f^{-1}=\operatorname{id}_{X}$ and $f^{-1} \circ f=\operatorname{id}_{Y}$. In particular, each $\operatorname{Mor}_{\mathfrak{G}}(X, X)$ is a group and we call it the vertex group at $X$.

If the set $\operatorname{Mor}(\mathfrak{G})$ of the morphisms is finite set, we call it finite. If $\operatorname{Mor}_{\mathfrak{G}}(X, Y) \neq \emptyset$ for any objects $X, Y \in \operatorname{Obj}(\mathfrak{G})$, we call it connected.

Proposition 6. [5, Proposition 12] All vertex groups of a connected groupoid $\mathfrak{G}$ are isomorphic.

Proof. Let $X, Y$ be any objects of $\mathfrak{G}$. Then, we have a morphism $g \in \operatorname{Mor}_{\mathfrak{G}}(X, Y)$ and the map $x \mapsto g^{-1} x g$ is a group isomorphism from the vertex group at $X$ to that of $Y$.

Thus, we call this group which is isomorphic to every vertex group the vertex group for the connected groupoid. If the connected groupoid $\mathfrak{G}$ is finite, the vertex group is a finite group.

Proposition 7. Let $X, Y$ be any two objects in a connected groupoid $\mathfrak{G}$. Then, $\operatorname{Mor}_{\mathfrak{G}}(X, Y)$ is regular as left $\operatorname{Mor}_{\mathfrak{G}}(X, X)$-sets. Similarly, $\operatorname{Mor}_{\mathfrak{G}}(X, Y)$ is regular as right $\operatorname{Mor}_{\mathfrak{G}}(Y, Y)$-sets.

Proof. We define the left action of $\operatorname{Mor}_{\mathfrak{G}}(X, X)$ to $\operatorname{Mor}_{\mathfrak{G}}(X, Y)$ by the composition of morphisms. Then, if we fix a morphism $\alpha \in$ $\operatorname{Mor}_{\mathfrak{G}}(X, Y)$, the map $f: \operatorname{Mor}_{\mathfrak{G}}(X, X) \rightarrow \operatorname{Mor}_{\mathfrak{G}}(X, Y)$ defined by $f(\beta)=\beta \alpha$ for any $\beta \in \operatorname{Mor}_{\mathfrak{G}}(X, X)$ is an isomorphism as $\operatorname{Mor}_{\mathfrak{G}}(X, X)$ set homomorphisms. We can obtain the desiring isomorphism similarly for the left action of $\operatorname{Mor}_{\mathfrak{G}}(Y, Y)$.

\section{Thin COHERENT CONFIGURATIONS}

In the present section, we will define the thin coherent configurations and investigate the properties and the structures of them.

Let $(X, C)$ be the coherent configuration. If we have that $|x g| \leq 1$ for any $x \in X, g \in C$, we say that $(X, C)$ is thin. This term is same as the term semi-regular in [4]. However, we may use the term thin from the following reason. If the coherent configuration $(X, C)$ is homogeneous, then $(X, C)$ is regarded as the association scheme. Moreover, if $(X, C)$ is thin also, then $(X, C)$ is the association scheme which is called thin in association scheme theory. 
In the rest of the present paper, we assume that the coherent configuration $(X, C)$ is thin. Now, since $\left(X_{f}, C_{f}\right)$ is homogeneous for any fiber $f \in C_{0},\left(X_{f}, C_{f}\right)$ is thin association scheme. Therefore, $\left(X_{f}, C_{f}\right)$ is essentially a finite group.

\subsection{From thin coherent configurations to finite connected groupoids.}

Let $(X, C)$ be a thin coherent configuration and $C_{0}$ be the fibers of $C$. We define the category $\mathfrak{G}$ as follows:

$$
\operatorname{Obj}(\mathfrak{G})=C_{0}, \operatorname{Mor}_{\mathfrak{G}}(f, g)={ }_{f} C_{g} \text { for any fibers } f, g \in C_{0} \text {. }
$$

Lemma 8. We have that $n_{c}={ }_{c} n=1$ for any $c \in C$. Moreover, we obtain that $\left|X_{f}\right|=\left|X_{g}\right|$ for any fibers $f, g \in C_{0}$

Proof. Since it follows that $n_{c} \geq 1$, we obtain that $n_{c}={ }_{c} n=1$ from the definition of thin coherent configurations. Thus, from Proposition 5, we have that $\left|X_{f}\right|=\left|X_{g}\right|$.

Lemma 9. Let $f, g$ and $h$ be fibers of $C$. There exists the unique element $e \in{ }_{f} C_{h}$ such that $A_{c} A_{d}=A_{e}$ for $c \in{ }_{f} C_{g}$ and $d \in{ }_{g} C_{h}$.

Proof. We set that $x \in X_{f}$. Since it follows that $|x c|=1$, there exists the element $y \in x c \subset X_{g}$. Moreover, since $|y d|=1$, there exists $z \in y d \subset X_{h}$. Thus, if we set that $(x, w) \in e$, it follows that $e \in{ }_{f} C_{h}$ and $p_{c d}^{e} \geq 1$. Then, we have that

$$
A_{c} A_{d}=\sum_{e \in f_{f} C_{g}} p_{e d}^{e} A_{e}=\sum_{e \in_{f} C_{g}} \frac{|c|}{|e|} p_{d e^{*}}^{c^{*}} A_{e}=\sum_{e \in_{f} C_{g}} p_{d e^{*}}^{c^{*}} A_{e} .
$$

However, we know from Proposition 5 that

$$
\sum_{e \in C_{f} C_{g}} p_{d e^{*}}^{c^{*}}=n_{d}=1
$$

Therefore, we have at most one element $e$ such that $p_{c d}^{e} \neq 0$. Thus we have the unique element $e \in{ }_{f} C_{g}$ such that $p_{c d}^{e}=1$.

From the above arguments, we can define the composition of morphisms by $s \circ t=u$ which satisfies the associative law. Moreover, we have the identity morphism $f$ of the object $f$ for any fiber $f \in C_{0}$ since $f \in{ }_{f} C_{f}$. Thus, $\mathfrak{G}$ is a category.

Theorem 10. The category $\mathfrak{G}$ is a finite connected groupoid.

Proof. We know that $c \circ c^{*}=f$ and $c^{*} \circ c=g$ for any $c \in{ }_{f} C_{g}$. Therefore it follows that $c$ is an isomorphism. Thus, $\mathfrak{G}$ is a groupoid. For $x \in X_{f}$ and $y \in X_{g}$, there exists the unique element $c \in C$ such that $(x, y) \in c$. So ${ }_{f} C_{g}=\operatorname{Mor}_{\mathfrak{G}}(f, g)$ is non-empty. Now $\mathfrak{G}$ is connected. 
3.2. From finite connected groupoids to thin coherent configurations. Let $\mathfrak{G}$ be a finite connected groupoids. We define a thin coherent configuration $(X, C)$ as follows:

We recall Propositions 6 and 7. Since $\mathfrak{G}$ is connected, $\operatorname{Mor}_{\mathfrak{G}}(x)$ is isomorphic to the vertex group $G$ for any $x \in \operatorname{Obj}(\mathfrak{G})$. We set that $G_{x}=\operatorname{Mor}_{\mathfrak{G}}(x)$ for all $x \in \operatorname{Obj}(\mathfrak{G})$. We will identity $G$ and $G_{x}$. We fix the morphism $\xi_{x_{0} x} \in \operatorname{Mor}_{\mathfrak{G}}\left(x_{0}, x\right)$ for all $x \in \operatorname{Obj}(\mathfrak{G})$. We assume that $\xi_{x_{0} x_{0}}=\mathrm{id}_{x_{0}}$.

For any object $x \in \operatorname{Obj}(\mathfrak{G})$, We define the map $\varphi_{x_{0} x}: G_{x_{0}} \rightarrow G_{x}$ by $\varphi_{x_{0} x}(g)=\xi_{x_{0} x}^{-1} \circ g \circ \xi_{x_{0} x}$. Then, it follows that $\varphi_{x_{0} x}$ is a group isomorphism. We set that $g^{(x)}=\varphi_{x_{0} x}(g)$ for any $g \in G_{x_{0}}$ and $x \in$ $\operatorname{Obj}(\mathfrak{G})$.

We define $\xi_{x y}=\xi_{x_{0} x}^{-1} \circ \xi_{x_{0} y}$ for all $x, y \in \operatorname{Obj}(\mathfrak{G})$. We obtain that $\xi_{x y}=\xi_{y x}^{-1}$ and $\xi_{x y} \circ \xi_{y z}=\xi_{x z}$ from definition. Moreover, we have that $g^{(x)} \circ \xi_{x y}=\xi_{x y} \circ g^{(y)}$ for any $x, y \in \operatorname{Obj}(\mathfrak{G})$.

Now, we will construct the thin coherent configuration $(X, C)$ from the finite connected groupoid $\mathfrak{G}$. We set that $X=\cup_{x \in \operatorname{Obj}(\mathfrak{G})} G_{x}$. We define the set $[\alpha]$ by

$$
[\alpha]=\left\{(m, n) \in G_{x} \times G_{y} \mid m \circ \xi_{x y} \circ n^{-1}=\alpha\right\} \text { for } \alpha \in \operatorname{Mor}_{\mathfrak{G}}(x, y) .
$$

Then we set $C=\{[\alpha] \mid \alpha \in \operatorname{Mor}(\mathfrak{G})\}$ and it follows that $C$ is the partition of $X \times X$.

Theorem 11. The configuration $(X, C)$ defined in the above argument is the thin coherent configuration. Moreover, $(X, C)$ is the direct product of the homogeneous coherent configuration obtained from the underlying group $G$ and the discrete coherent configuration of degree $|\operatorname{Obj}(\mathfrak{G})|$.

Proof. We have the labeling for the elements of $G_{x}$ by the elements of $G_{x_{0}}$. Namely, the element $g^{(x)} \in G_{x}$ is corresponding to the element $g \in$ $G_{x_{0}}$. Similarly, we can obtain the labeling the elements of $\operatorname{Mor}_{\mathfrak{G}}(x, y)$ by the elements of $G_{x_{0}}$ as follows. Now, we know that $\operatorname{Mor}_{\mathfrak{G}}(x, y)=$ $G_{x} \circ \xi_{x y}$. Moreover, since $\operatorname{Mor}_{\mathfrak{G}}(x, y)$ is a regular $G_{x}$-set, there exists the unique description for $\operatorname{Mor}_{\mathfrak{G}}(x, y)$ such that $\operatorname{Mor}_{\mathfrak{G}}(x, y)=\left\{g^{(x)} \circ \xi_{x y} \mid\right.$ $\left.g \in G_{x_{0}}\right\}$. Here, for $f, g, h \in G_{x_{0}}$ and $x, y, z \in \operatorname{Obj}(\mathfrak{G})$, we have

$$
\begin{aligned}
\left(f^{(x)}, g^{(x)}\right) \in\left[h^{(x)}\right] & \Leftrightarrow f g^{-1}=h \\
& \Leftrightarrow f^{(y)} \circ \xi_{y z} \circ\left(g^{(z)}\right)^{-1}=h^{(y)} \circ \xi_{y z} \\
& \Leftrightarrow\left(f^{(y)}, g^{(z)}\right) \in\left[h^{(y)} \circ \xi_{y z}\right]
\end{aligned}
$$

This means that the partition of $G_{y} \times G_{z}$ has the same structure for any $y, z \in \operatorname{Obj}(\mathfrak{G})$. Thus, $(X, C)$ is the direct product of the homogeneous coherent configuration obtained from the vertex group $G$ and the discrete coherent configuration of degree $|\mathrm{Obj}(\mathfrak{G})|$. 
3.3. Conclusion and Remarks. We have the following conclusion.

Theorem 12. A thin coherent configuration defines a finite connected groupoid. Conversely, a finite connected groupoid defines a thin coherent configuration.

By our arguments, we also have the following statement. It seems to be known for specialists.

Theorem 13. Let $(X, C)$ be a thin coherent configuration and $C_{0}$ be the set of fibers of $C$. Then, all fiber schemes are isomorphic to each other and essentially finite groups. Moreover, $(X, C)$ is isomorphic to the direct product of a fiber scheme and the discrete coherent configuration of degree $\left|C_{0}\right|$.

We fix a fiber $f \in C_{0}$. The fiber scheme $\left(X_{f}, C_{f}\right)$ is essentially a finite group $G$. Let $F$ be any field. Then, the adjacency algebra $F C_{f}$ of the fiber scheme $\left(X_{f}, C_{f}\right)$ over the field $F$ is isomorphic to the group algebra $F G$ of the finite group $G$. On the other hand, the adjacency algebra of the discrete coherent configuration of degree $n$ over $\mathrm{F}$ is isomorphic to the matrix algebra $M_{n}(F)$. Thus, we have the following theorem. For example, see $[3, \S 3 \mathrm{D}]$.

Theorem 14. Let $(X, C)$ be a thin coherent configuration and $C_{0}$ be the set of fibers of $C$. We set that the finite group $G$ is corresponding to a fiber scheme $\left(X_{f}, C_{f}\right)$. Then, for any field $F$,

$$
F C \cong F G \otimes_{F} M_{\left|C_{0}\right|}(F) \cong M_{\left|C_{0}\right|}(F G) .
$$

Therefore, the module category of FC is Morita equivalent to the module category of $F G$.

Finally we list some remarks.

Remark 15. When we construct the coherent configuration from the groupoid, the partition of $X \times X$ is depend on the choice of $\xi_{x_{0} x} \in$ $\operatorname{Mor}_{\mathfrak{G}}\left(x_{0}, x\right)$. However, the coherent configurations from the same groupoid are isomorphic to each other.

Remark 16. The corresponding between thin coherent configurations and finite connected groupoids is one to one up to isomorphic.

Remark 17. For a disconnected finite groupoid, we can define the coherent configuration (not necessary to be thin) as follows. If we have $\operatorname{Mor}_{\mathfrak{G}}(x, y) \neq \emptyset$, we define the relations as the same as the case of connected. Otherwise, we define the relation which is whole $G_{x} \times G_{y}$. Thus we obtain the coherent configuration from the disconnected finite groupoid. 


\section{REFERENCES}

[1] E. Bannai and T. Ito, "Algebraic Combinatorics. I. Association Schemes," Benjamin-Cummings, Menlo Park, CA, 1984.

[2] A. E. Brouwer, A. M. Cohen and A. Nuemaier, "Distance-Regular Graphs" Springer-Verlag, Berlin, Heidelberg, 1989.

[3] C. W. Curtis and I. Reiner, "Methods of representation theory. Vol. I" John Wiley \& Sons, New York, 1981.

[4] S. Evdokimov and I. Ponomarenko, Separability Number and Schurity Number of Coherent Configurations,

[5] P. J. Higgins, "Notes on categories and groupoids," ,Van Nostrand Rienhold Mathematical Studies, No. 32, Van Nostrand Reinhold Co., 1971.

[6] D. G. Higman, Coherent configurations. I. Part I: Ordinary representation theory, Geom. Dedicata 4, 1-32 (1975).

[7] P. -H. Zieschang, "An Algebraic Approach to Association Schemes", Lecture Notes in Math. vol. 1628, Springer, Berlin-Heidelberg-New York, 1996.

[8] P. -H. Zieschang, "Theory of Association schemes", Springer Monographs in Mathematics, Berlin-Heidelberg-New York, 2005.

(A. Hanaki) Department of Mathematical Sciences, Faculty of Sciences, Shinshu University, Matsumoto, Nagano, 390-8621, Japan

Email address: hanaki@shinshu-u.ac.jp

(M. Yoshikawa) Nagano Prefecture Iida Fuetsu Senior High School, 6462 Kamisato Kuroda, Iida, Nagano, 395-8543, Japan

Email address: yosikawa@nagano-c.ed.jp 